\title{
MESHFREE MODELLING OF DYNAMIC FRACTURE IN FIBRE REINFORCED CONCRETE
}

\author{
RENA C. YU* ${ }^{*}$ PEDRO NAVAS ${ }^{\dagger}$ AND GONZALO RUIZ \\ *,\$Universidad de Castilla-La Mancha (UCLM) \\ Avda. Camilo José Cela s/n, 13071 Ciudad Real, Spain \\ e-mail: rena@uclm.es, Gonzalo.Ruiz@uclm.es \\ $\dagger$ Universidad Politécnica de Madrid \\ Calle Profesor Aranguren, 28040 Madrid, Espaã \\ e-mail: Pedro.Navas@upm.es
}

Key words: Cohesive fracture, Fiber Reinforced Concrete, Meshfree modelling

\begin{abstract}
This work is concerned with the numerical study of dynamic mode I and mixedmode fracture in fiber reinforced concrete (FRC). A recently developed eigensoftening algorithm to deal with the fracture of quasi-brittle materials is employed in a meshfree framework. Threepoint bending tests on notched beams reinforced with steel fibers carried out through a drop weight device at two loading velocities are modelled herein. Since the notch was placed with an offset from the middle section, mixed-mode crack formation was facilitated. Three types of concrete with the same matrix reinforced with different amounts of steel fibers were used for these beams. All mechanical and fracture properties were measured through independent tests. Assuming a linear softening stress-equivalent crack opening relation, the numerical methodology is first validated against experimental results on plain concrete. Subsequently, it is applied to study the dynamic fracture of fiber reinforced concrete with a bilinear softening relation. The numerical simulations reproduced remarkably well the experimental results such as load-line displacements, crack patterns and reaction forces. The parametric studies show that the total energy dissipation plays an important role on the peak reaction load, whereas the transitional point between the two branches has a significant influence on the crack patterns.
\end{abstract}




\section{INTRODUCTION}

The rate effect on fracture propagation in concrete with or without reinforcements has been the centre of interest for the last three decades, from both experimental and numerical standpoints. In the current work, we endeavor to model the dynamic fracture propagation (mode I and mixed-mode) in plain and fiber reinforced concrete employing the optimal transportation meshfree (OTM) approximation scheme [1, 2, 3, 4]. A concept based on the eigenerosion approach to brittle fracture developed by Pandolfi et al. [5], the eigensoftening procedure is proposed for quasi-brittle materials [6]. This is engineered for the gradual rather than abrupt dissipation of the fracture energy. In other words, the material softens in a progressive manner until its complete failure, i.e. the formation of a stress-free crack. This is equivalent to the crack band model [7], since energy dissipation is through the softened (or failed) volume. In the meantime, the analogy to cohesive approaches is straightforward through the definition of a damage variable. Furthermore, the implementation follows the same procedure as that of the eigenerosion algorithm except for the strength criterion for crack initiation. Consequently, a simple alternative particularly suitable for a meshfree framework, is formulated for the fracture quasi-brittle materials. The current work is a short version of the work developed in detail in [6, 8].

Next the experimental setup and observations are presented. The meshfree methodology, eigenerosion and eigensoftening algorithms for fracture are described in Section 3. Numerical results are given in Section 4. Finally, relevant conclusions are drawn in Section 5 .

\section{EXPERIMENTAL OBSERVATIONS}

While the experimental work on the rate effect on flexural fracture in high strength concrete [9, 10], the experimental campaign on the dynamic mixed-mode fracture of plain
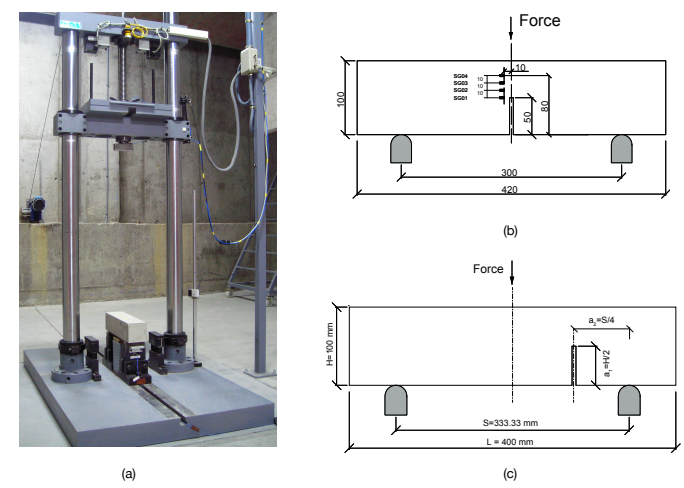

Figure 1: (a) The drop-weight device developed by Zhang et al. [11; (b) three-point bend beam with a central notch to facilitate mode-I fracture propagation; (c) three-point bend beam with an offset notch to facilitate mixed-mode fracture initiation.

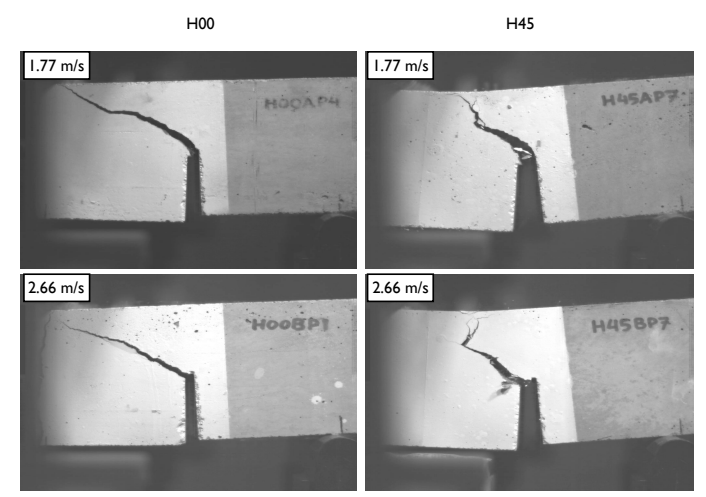

Figure 2: Typical crack patterns for beams with an offset notch made from plain concrete (H00) and concrete reinforced with $45 \mathrm{~kg} / \mathrm{m}^{3}$ steel fibres (H45) impacted at $1.77 \mathrm{~m} / \mathrm{s}$ (top row) and $2.66 \mathrm{~m} / \mathrm{s}$ (bottom row).

and steel fiber reinforced concrete by Ruiz et al. [12 was designed to study the influence of loading velocity and fiber content on the developed crack patterns.

The drop-weight device [11 designed and constructed in the Laboratory of Materials and Structures at the University of CastillaLa Mancha was employed for exerting different loading velocities (in particular 881, 1770 and $2660 \mathrm{~mm} / \mathrm{s}$ ) on notched beams by dropping a mass up to $120.6 \mathrm{~kg}$. The impact force was measured through a piezoelectric force sensor and the reaction force was determined by two force sensors located between the supports and the specimen. For mixedmode fracture, beams of $100 \times 100 \mathrm{~mm}^{2}$ in 
cross section, $400 \mathrm{~mm}$ in length, $333 \mathrm{~mm}$ in span, see Fig. 1, were tested in a three-point bend configuration. The notch is located at an offset of a quarter span from the central section. The initial notch-depth ratio was approximately 0.5 .

In the experimental campaign [12], concrete with the same matrix but reinforced with different fiber contents was tested. For instance, H0O is the base concrete, fabricated as a reference material, whereas $\mathrm{H} 15$ and $\mathrm{H} 45$ are with the same matrix but reinforced with 15 and $45 \mathrm{~kg} / \mathrm{m}^{3}$ of steel fibers. The fibers were $35 \mathrm{~mm}$ in length and $0.55 \mathrm{~mm}$ in diameter. Material properties, such as the elastic modulus, E, the Poisson's ratio, the compressive strength, $f_{c}$, the tensile strength, $f_{t}$ and the specific fracture energy, $G_{F}$, were all characterized with independent tests.

Typical crack patterns loaded at two different velocities are given in Fig.2 for the base concrete $\mathrm{H} 00$ and the concrete reinforced with $45 \mathrm{~kg} / \mathrm{m}^{3}$ of steel fibers, H45. Note that as the increase of fiber content, the crack is going away from the loading zone. In addition, as the compressive zone is approached, crack bifurcation occurs.

\section{NUMERICAL METHODOLOGY}

\subsection{The OTM scheme}

The OTM approach [1] is a meshfree updated Lagrangian numerical scheme that combines concepts from optimal transportation theory with a material-point sampling and the local max-ent meshfree approximation [13]. The method has been used in applications involving dynamic deformation and failure of materials with excellent results. The numerous advantages previously mentioned ensure its robustness and stability in numerical calculations.

Two sets of points, namely, nodal points and material points are introduced in the OTM method. A schematic of the OTM approximation scheme for an incremental motion from time $t_{k}$ to $t_{k+1}$ is shown in Fig. 3 . The kinematic information of the domain,

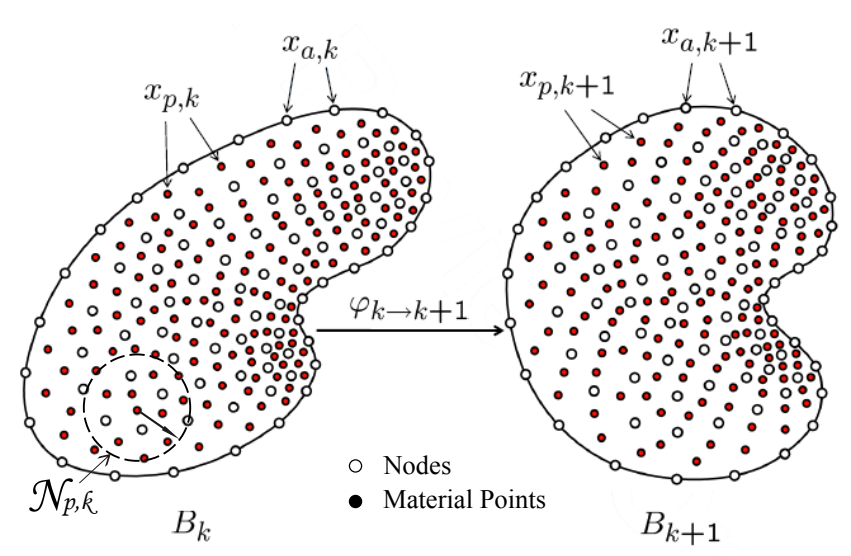

Figure 3: The incremental motion from time $t_{k}$ to $t_{k+1}$ in the OTM approximation scheme. The empty circles represent the nodal points, $x_{a, k}$ and $x_{a, k+1}$, whereas solid circles stand for material points $x_{p, k}$ and $x_{p, k+1}$.

such as the displacement, velocity and acceleration field, is stored at the nodes. The material responses and local state variables are evaluated at the material points, which are also transported by the incremental motion of the domain. To approximate the incremental motion from nodal displacement field, the local max-ent approximation scheme defined by Arroyo and Ortiz 13 is employed. The local max-ent function (LME), as a Pareto set, is optimal for $\beta \in(0, \infty)$ and obtained as

$$
N_{a}(\mathbf{x})=\frac{\exp \left[-\beta\left|\mathbf{x}-\mathbf{x}_{\mathbf{a}}\right|^{2}+\boldsymbol{\lambda}^{*} \cdot\left(\mathbf{x}-\mathbf{x}_{\mathbf{a}}\right)\right]}{Z\left(\mathbf{x}, \boldsymbol{\lambda}^{*}(\mathbf{x})\right)},
$$

where

$Z(\mathbf{x}, \boldsymbol{\lambda})=\sum_{a=1}^{n} \exp \left[-\beta\left|\mathbf{x}-\mathbf{x}_{\mathbf{a}}\right|^{2}+\boldsymbol{\lambda} \cdot\left(\mathbf{x}-\mathbf{x}_{\mathbf{a}}\right)\right]$,

being $\boldsymbol{\lambda}^{*}(\mathrm{x})$ the unique minimizer for $\log Z(\mathbf{x}, \boldsymbol{\lambda})$. The parameter $\beta$ is related with the discretization size (or nodal spacing), $h$, whereas $\gamma$ controls the locality of the shape functions, as follows

$$
\beta=\frac{\gamma}{h^{2}} \text {. }
$$

For a uniform nodal spacing, $\beta$ is also a constant, thus the first derivatives can be obtained by employing the following expression

$$
\nabla N_{a}^{*}=-N_{a}^{*}\left(\mathbf{J}^{*}\right)^{-1}\left(\mathbf{x}-\mathbf{x}_{\mathbf{a}}\right)
$$


where $\mathbf{J}$ is the Hessian matrix, defined by

$$
\begin{aligned}
\mathbf{J}(\mathbf{x}, \boldsymbol{\lambda}, \beta) & =\frac{\partial \mathbf{r}}{\partial \boldsymbol{\lambda}} \\
\mathbf{r}(\mathbf{x}, \boldsymbol{\lambda}, \beta) & \equiv \partial_{\boldsymbol{\lambda}} \log Z(\mathbf{x}, \boldsymbol{\lambda}) \\
& =\sum_{a} N_{a}(\mathbf{x}, \boldsymbol{\lambda}, \beta)\left(\mathbf{x}-\mathbf{x}_{\mathbf{a}}\right) .
\end{aligned}
$$

Note that the objective of the above procedure is to find the $\boldsymbol{\lambda}$ which minimizes $\log Z(\mathbf{x}, \boldsymbol{\lambda})$. This unconstrained minimization problem with a strictly convex objective function can be solved efficiently and robustly by a combination of the Newton-Raphson method and Nelder-Mead Simplex algorithm [14].

In calculations, the max-ent shape functions are reconstructed continuously from the nodal set, which changes in every step by adapting to the local deformation of the material points. This overcomes the essential difficulties of mesh entanglement that arise in Lagrangian grid-based numerical schemes. On the other hand, material points result from the spatial approximation of the mass densities $\rho_{h, k}(x)$ by $M$ points

$$
\rho_{h, k}(x)=\sum_{p=1}^{M} m_{p} \delta\left(x-x_{p, k}\right),
$$

where $x_{p, k}$ represents the position at time $t_{k}$ of material point $p, m_{p}$ is the mass of the material point and $\delta\left(x-x_{p, k}\right)$ is the Dirac-delta distribution centered at $x_{p, k}$. Material points are convected by the deformation and carry a fixed mass as well as serve the purpose of integration points for the calculation of the effective nodal forces and masses. The spatial discretization is completed by approximating the deformation mapping as

$$
\varphi_{h, k \rightarrow k+1}(x)=\sum_{a=1}^{N} x_{a, k+1} N_{a, k}(x)
$$

where $x_{a, k+1}$ is the position of node $a$ at time $t_{k+1}$, and $N_{a, k}(x)$ are max-ent shape functions defined over the configuration for node $a$ at time $t_{k}$. The interpolation at a material point $x_{p, k}$ depends solely on the nodes contained in a small local neighborhood $N_{p, k}$ as shown in Fig. 3. The reconstruction of the local neighborhoods leads to a new reconnection of the material points and the nodal set on the fly adaptively.

\subsection{The eigensoftening algorithm for fracture}

The eigensoftening algorithm was proposed by Navas et al. [6] as an extension of the eigenerosion approach by Pandolfi et al. [3, 4 ] to quasi-brittle fracture. It was validated against experimental tests for the dynamic mode-I fracture in high strength concrete. Herein, the methodology is applied to study the dynamic mixed-mode fracture of steel fiber reinforced concrete.

The implementation of the eigensoftening algorithm is based on a strength criterion for the crack initiation and a softening law which is proper to the material under study before the formation of a stress-free crack. For the stress measurement, the maximum principal stress theory is considered for brittle fracture at the current time $t_{k+1}$ for the material point $q$. In other words, $\sigma_{q, 1}$ is the maximum principal stress for material point. Consequently, the definition of an equivalent critical stress at the material point $x_{p, k+1}$ for a $B_{\epsilon}$ neighborhood can be calculated as follows

$$
\begin{aligned}
\sigma_{p}^{\epsilon} & =\frac{1}{m_{p}} \sum_{x_{q, k+1} \in B_{\epsilon}\left(x_{p, k+1}\right)} m_{q} \sigma_{q, 1} \\
m_{p} & =\sum_{x_{q, k+1} \in B_{\epsilon}\left(x_{p, k+1}\right)} m_{q} .
\end{aligned}
$$

The concept of the $B_{\epsilon}$-neighborhood was utilized for the eigenerosion algorithm by Pandolfi and Ortiz [5]. In this neighborhood, the material points involved in the fracture process are located. A parametric study confirmed that the size of this neighborhood for accurate results yields between one and two times the nodal spacing.

For a quasi-brittle behavior, when $\sigma_{p, k+1}^{\epsilon}$ surpasses the tensile strength, $f_{t}$, the softening law is activated, which in turn causes a 
reduction of the internal forces as follows,

$$
f^{i n t}=(1-\chi) \sum^{p} \int_{\Omega} \sigma_{p, k+1} \nabla N d V,
$$

where $\chi$ is the damage variable, ranges between zero (an intact material) and one (completely failed material points).

In addition, the softening process is governed by a stress-crack opening displacement relation, often termed as cohesive law. For the eigensoftening algorithm, a length scale, $h^{\epsilon}$, equivalent to the crack band model of Bažant [7], is defined. A reference value for $h^{\epsilon}$ between two and four times the maximum size of the aggregates for concrete was recommended by Bažant [7]. The effective fracture strain, $\varepsilon_{f}^{\epsilon}$, defined as the difference between the maximum principal strain at crack initiation, $\varepsilon_{1}\left(x_{p, 0}\right)$, and the current one, $\varepsilon_{1}\left(x_{p, k+1}\right)$, for material point $p, k+1$ being the current integration time. Meanwhile, $\varepsilon_{f}^{\epsilon}$ can also be represented as the current crack opening displacement, $w$, within the band width, $h^{\epsilon}$. Consequently, for a linear softening behaviour,

$$
\varepsilon_{f}^{\epsilon}(w)=\varepsilon_{1}\left(x_{p, k+1}\right)-\varepsilon_{1}\left(x_{p, 0}\right)=\frac{w}{h^{\epsilon}} .
$$

Therefore, the damage variable is pertinent to cohesive law parameters such as the fracture energy, $G_{F}$, the critical crack opening displacement, $w_{c}$ and the tensile strength, $f_{t}$, as well as the effective fracture strain and the crack band width.

$$
\chi=\chi\left(\varepsilon_{f}^{\epsilon}, h^{\epsilon}, f_{t}, w_{c}, G_{f}, w\right) .
$$

In Fig, 4 , the influence of the band width parameter, $h^{\epsilon}$, is demonstrated for a modeI crack propagation in a three-point bend beam loaded at $2.66 \mathrm{~m} / \mathrm{s}$. The maximum aggregate size of the material is of $12 \mathrm{~mm}$. The stress distribution is demonstrated for three values of $h^{\epsilon}, 12,30$ and $60 \mathrm{~mm}$ when the peak load is attained in Fig, 4 a. Note that when the band width is too small, the stresses at the crack tip are overestimated,

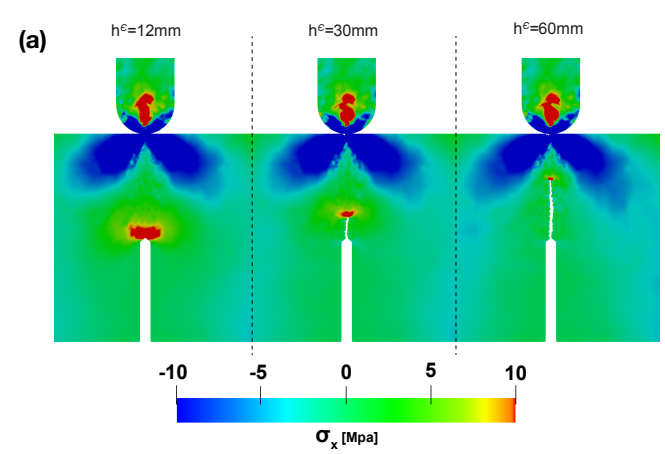

(b)

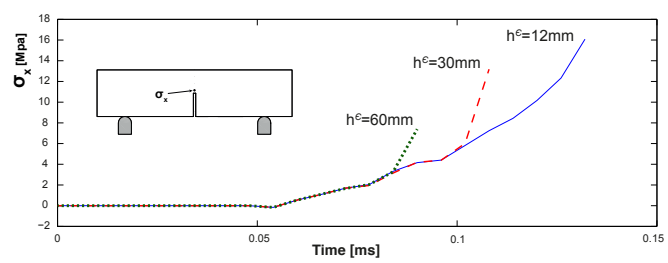

Figure 4: Influence of the band width parameter: (a) stress distribution at peak load; (b) stress evolution for a point above the notch point for a three-point beam impacted at $2.66 \mathrm{~m} / \mathrm{s}$.

consequently the crack initiation is delayed, the crack propagation takes longer time, as can be seen in Fig $4 \mathrm{~b}$, where the stress evolution for a point above the notch tip is compared for three values of the band width.

\section{DYNAMIC MIXED-MODE FRAC- TURE IN PLAIN AND FIBER RE- INFORCED CONCRETE}

\subsection{Softening law for fiber reinforced concrete}

In order to model the fracture behavior of fiber reinforced concrete, as a first approximation, the fracture anergy of the base concrete is augmented by a factor $\eta$, i.e. $G_{F_{2}}=$ $\eta G_{F_{1}}$. For a linear softening law, the parameter $\eta$ is equal to the amplification factor, $\alpha$, for the critical opening displacement of the base concrete, $w_{c}$, see Fig. 5. For a bilinear relation, the transition point between the linear segments need to be calibrated. For both cases, the contribution of the base concrete is through the linear-decreasing cohesive law,

$$
\sigma_{b}(w)=f_{t}\left(1-\frac{w}{w_{c}}\right)
$$




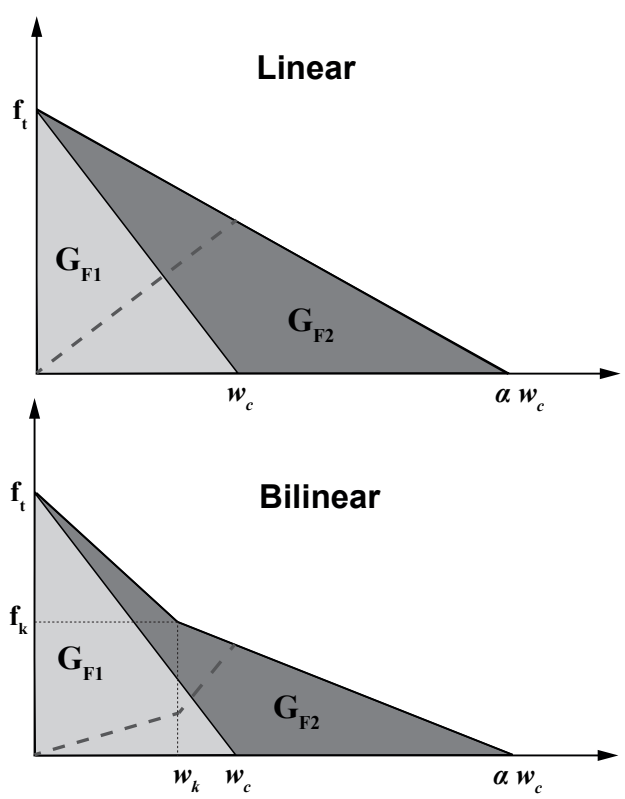

Figure 5: Linear and bilinear cohesive laws for the FRC, where the lightly-shaded area is the specific fracture energy, $G_{F_{1}}, f_{t}$ is the tensile strength, and $w_{c}$ is the critical opening displacement of the base concrete; whereas $G_{F_{2}}$ is the equivalent specific fracture for FRC. The dashed lines outline the fiber contribution.

whereas the fiber contribution, activated once the crack is open, is obtained as

$$
\sigma_{f}(w)=\sigma(w)-\sigma_{b}(w) .
$$

Subsequently, the damage variable is calculated as:

$$
\chi(w)=1-\frac{\sigma(w)}{f_{t}}
$$

\subsection{Simulated crack patterns}

First, the developed methodology is applied to simulate the mixed-mode fracture propagation in a plain concrete (H00) beam. The simultaneous flexural and shear crack propagation is observed, see Fig 6 for the damage distribution at $0.17,0.19$ and $0.20 \mathrm{~ms}$ after the beam was impacted at $2.66 \mathrm{~m} / \mathrm{s}$. Next, employing a bilinear softening relation, with different amplification factors for $G_{F}$, the mixed-mode fracture is reproduced for $\mathrm{H} 00, \mathrm{H} 15$ and $\mathrm{H} 45$ beams impacted at 1.77 and $2.66 \mathrm{~m} / \mathrm{s}$, respectively. The comparison

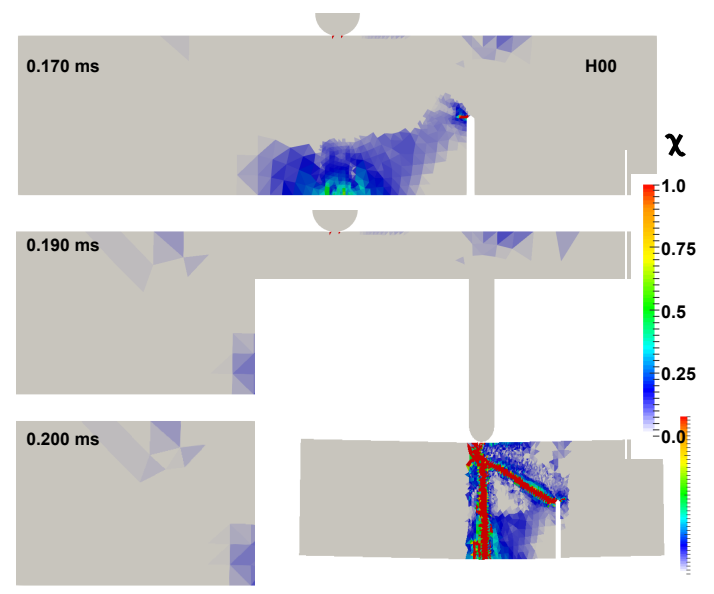

Figure 6: Damage evolution for the simultaneous flexural and shear crack propagation for H0O impacted at $2.66 \mathrm{~m} / \mathrm{s}$.

with the experimental crack patterns is illustrated in Fig. 7b. Additionally given in Fig. $7 \mathrm{a}$ is the distribution of the damage variable $\chi$ for a flexural beam impacted at 0.881 , 1.77 and $2.66 \mathrm{~m} / \mathrm{s}$. Note that crack bifurcation is observed near the impact point for higher loading velocities.

\section{CONCLUSIONS}

A recently developed eigensoftening algorithm is employed to study the dynamic fracture in mixed-mode in plain and fiber reinforced concrete under two impact velocities. The calculations were carried out in a meshfree framework. Three point bending beams with notches with an offset from the middle section were modelled. Even though a notch away from the middle section facilitated the initiation of flexural cracks, two main cracks were formed in plain concrete beams, whereas the crack started from the notch tip developed into a macro crack when fibers were added. The numerical simulations reproduced very well these crack patterns.

\section{Acknowledgements}

The financial support to develop this research from the Ministerio de Ciencia e Innovación, under Grant Number BIA2015-686 $78-\mathrm{C} 2-1-\mathrm{R}$ is greatly appreciated. 
(a)
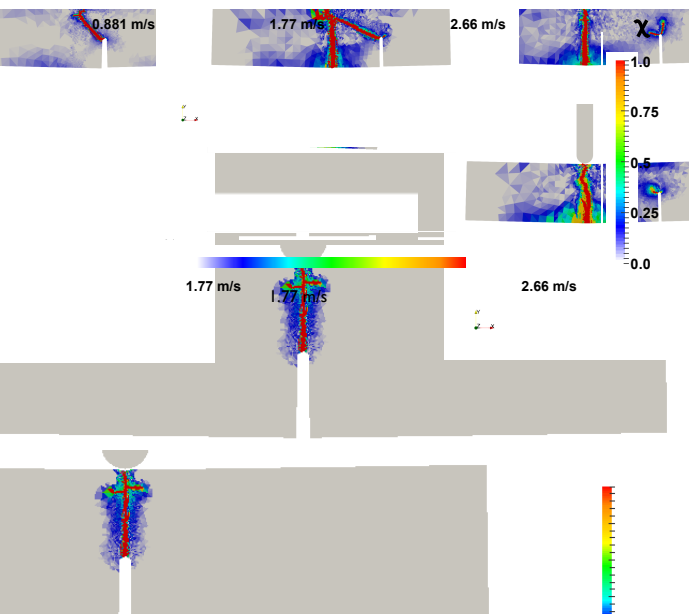

Figure 7: Simulated crack patterns: (a) mode I: central-notched beams impacted at $0.881,11.77$ and $2.66 \mathrm{~m} / \mathrm{s}$, respectively; (b) mixed-mode: offsetnotched beams made from H00, H15 and H45 impacted at 1.77 and $2.66 \mathrm{~m} / \mathrm{s}$, respectively.

\section{REFERENCES}

[1] B. Li, F. Habbal, M. Ortiz, Optimal transportation meshfree approximation schemes for fluid and plastic flows, Int. J. Numer. Methods Eng. 83 (2010) 1541-1579.

[2] B. Li, A. Kadane, G. Ravichandran, M. Ortiz, Verification and validation of the optimal-transportation meshfree (OTM) simulation of terminal ballistics, Int. J. Impact Eng. 42 (2012) 25-36.

[3] A. Pandolfi, B. Li, M. Ortiz, Modeling fracture by material-point erosion, Int. J. of Fract. 184 (2013) 3-16.

[4] B. Li, A. Pandolfi, M. Ortiz, Materialpoint erosion simulation of dynamic fragmentation of metals, Mech Mater 80 (2015) 288-297.

[5] A. Pandolfi, M. Ortiz, An eigenerosion approach to brittle fracture, Int. J. Numer. Methods Eng. 92 (2012) 694-714.

[6] P. Navas, R. C. Yu, B. Li, G. Ruiz, Modeling the dynamic fracture in concrete: an eigensoftening meshfree approach, Int. J. Impact Eng. 113 (2018) 9-20.
[7] Z. Bažant, B. Oh, Crack band theory for fracture in concrete, Mater. Struct. 16 (1983) 155-177.

[8] R. C. Yu, P. Navas, G. Ruiz, Meshfree modeling of the dynamic mixed-mode fracture in FRC through an eigensoftening approach, Eng. Struct. 172 (2018) 94-104.

[9] X. X. Zhang, G. Ruiz, R. C. Yu, M. Tarifa, Fracture behaviour of high-strength concrete at a wide range of loading rates, Int. J. Impact. Eng. 36 (10-11) (2009) 1204-1209.

[10] X. X. Zhang, R. C. Yu, G. Ruiz, M. Tarifa, M. Camara, Effect of loading rate on crack velocities in HSC, Int. J. Impact. Eng. 37 (4) (2010) 359-370.

[11] X. X. Zhang, G. Ruiz, R. C. Yu, A new drop-weight impact machine for studying fracture processes in structural concrete, Strain 46(3) (2010) 252-257.

[12] G. Ruiz, A. de la Rosa, L. C. Almeida, E. Poveda, X. X. Zhang, M. Tarifa, Z. M. Wu, R. C. Yu, Dynamic mixedmode fracture in SCC reinforced with steel fibers: an experimental study, Int. J. Impact. Eng. 129 (2019) 101-111.

[13] M. Arroyo, M. Ortiz, Local maximumentropy approximation schemes: a seamless bridge between finite elements and meshfree methods, Int. J. Numer. Methods Eng. 65 (13) (2006) 2167-2202.

[14] P. Navas, S. López-Querol, R. Yu, B. Li, B-bar based algorithm applied to meshfree numerical schemes to solve unconfined seepage problems through porous media, Int. J. Numer. Anal. Meth. Geomech. 40 (2016) 962-984. 